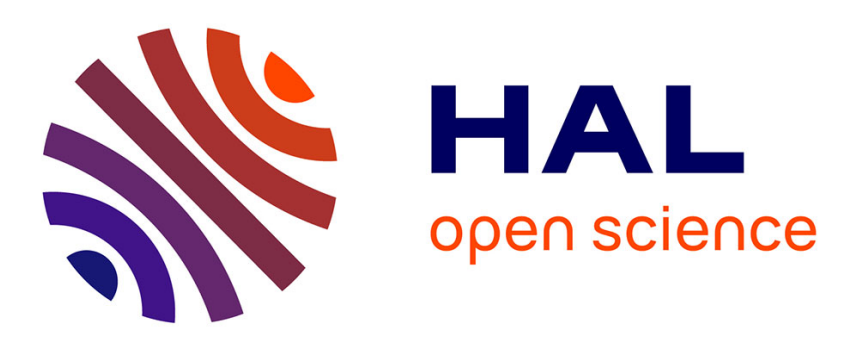

\title{
Mathematical modeling of an urban pigeon population subject to local management strategies
}

\author{
Ihab Haidar, Isabelle Alvarez, Anne-Caroline Prévot
}

\section{To cite this version:}

Ihab Haidar, Isabelle Alvarez, Anne-Caroline Prévot. Mathematical modeling of an urban pigeon population subject to local management strategies. Mathematical Biosciences, 2017, 288, pp.71-83. 10.1016/j.mbs.2017.03.002 . hal-01319753

\section{HAL Id: hal-01319753 \\ https://hal.sorbonne-universite.fr/hal-01319753}

Submitted on 23 May 2016

HAL is a multi-disciplinary open access archive for the deposit and dissemination of scientific research documents, whether they are published or not. The documents may come from teaching and research institutions in France or abroad, or from public or private research centers.
L'archive ouverte pluridisciplinaire HAL, est destinée au dépôt et à la diffusion de documents scientifiques de niveau recherche, publiés ou non, émanant des établissements d'enseignement et de recherche français ou étrangers, des laboratoires publics ou privés. 


\title{
Mathematical modeling of an urban pigeon population subject to local management strategies
}

\author{
I. Haidar ${ }^{\mathrm{a}, \mathrm{b}, \mathrm{d}, *}$, I. Alvarez ${ }^{\mathrm{b}, \mathrm{c}}$, A.C. Prévot ${ }^{\mathrm{d}}$ \\ ${ }^{a}$ Sorbonne Universités, UPMC Univ Paris 06, Institut de Mathématiques de Jussieu, 4 place Jussieu, \\ 75005, Paris, France. \\ ${ }^{b}$ Sorbonne Universités, UPMC Univ Paris 06, UMR 7606, LIP6, F-75005, Paris, France \\ ${ }^{c}$ Irstea, LISC, Aubière, France. \\ ${ }^{d}$ Centre d'Écologie et des Sciences de la Conservation (CESCO UMR 7204), Sorbonne Universités, \\ MNHN, CNRS, UPMC, CP135, 57 rue Cuvier, 75005, Paris, France
}

\begin{abstract}
This paper addresses the issue of managing urban pigeon population using some possible actions that makes it reach a density target with respect to socio-ecological constraints. A mathematical model describing the dynamic of this population is introduced. This model incorporates the effect of some regulatory actions on the dynamic of this population. We then used mathematical viability theory, which provides a framework to study compatibility between dynamics and state constraints. The viability study shows when and how it is possible to regulate the pigeon population with respect to the constraints.
\end{abstract}

Keywords: Biodiversity governance, Pigeons, Viability theory, Dynamical system.

\section{Introduction}

Urban pigeon Columba livia populations can reach high densities in cities and cause cohabitation problems with urban citizens (see e.g. Jerolmack (2008)). In response to social complains, different regulation programs are provided by local authorities to reduce perceived nuisance and help the coexistence between city dwellers and urban pigeons. These programs include different measures, from culling young or adult pigeons, to more welfarebased approaches (see e.g. Haag-Wackernagel (2002)). One example is the building of public pigeon houses where food and nest-sites are provided for pigeons and where most laid eggs are removed or sterilized (see e.g. Jacquin et al. (2010)). Complementarily, in order to control food resources for pigeons, pigeon feeding has been banned in most large cities. However, these regulation methods have not been shown to successfully reduce pigeon numbers (except in Basel, Haag-Wackernagel (1993)). In contrast, the so-called "pigeon problem" actually combines ecological and sociological issues (see e.g. Skandrani et al. (2014)): human-pigeon coexistence is not solely a function of pigeon numbers (and thus based on pigeon population

\footnotetext{
${ }^{*}$ Corresponding author. Email: Ihab.HAIDAR@imj-prg.fr
} 
control), but could be considered, as proposed in Skandrani et al. (2015), more successfully in terms of resilience and public perceptions of pigeons.

A successful regulation strategy requires a minimum of knowledge about the pigeons ecology and the ecological consequences of any regulation method. In fact, experimental evidences showed long-term side effects of some regulation methods implemented by local authorities (see e.g. Giunchi et al. (2007); Jacquin et al. (2010)). For example, egg removal may lead to a decrease in adult pigeon's body condition and egg quality of pigeons, as well as an increase in laying frequency and the total yearly number of laid eggs. Indeed, in Jacquin et al. (2010), the authors explored the consequences of repeated egg removal on egg-laying cycles and egg quality of feral pigeons breeding in pigeon houses. During four years, they compared the egg quality and egg-laying cycles of pigeons breeding in different pigeon houses which are subject to egg removal strategy to that of another population without egg removal. They observed that in pigeon houses with egg removal, the laying cycles were three times shorter than that in the pigeon houses without egg removal. Regarding direct regulation of population size, the study of genetic structures of pigeons population strongly suggests pigeons can disperse from one site to another (see e.g. Jacob et al. (2014)); this confirms empirical observations that reducing the number of pigeons in a particular site (whatever the method) is followed by the arrival of new individuals. Finally, as argued in Sacchi et al. (2002), limiting breeding resources neither seems guaranteeing limitation in pigeon number. The "pigeon problem" appears to be unsolvable by considering one single ecological variable.

The purpose of this study is to propose a model describing the evolution of a given fictive urban pigeon population impacted by regulation programs, by taking into account the different side effects highlighted in the literature (see above). In more detail, we model the dynamics of an urban pigeon population which is subject to two different regulation strategies: egg removal and food resource limitation. We model urban citizen satisfaction through the tolerance of a given maximum number of pigeons in a particular site. The ecological considerations are modeled through the requirement of a given minimum number of pigeons. Here, we explore a way to consider the trade-off between ecological considerations and urban citizen satisfaction. In other words, we explore how to maintain a given pigeon population under desirable constraints. Furthermore, we consider that this pigeon population is split into two sub-populations in two different sites. Because urban citizen satisfaction may differ from one site to another, we suppose that in each site the pigeon population is subject to different egg removal and limiting food resources strategies. According to pigeons ecology, we also consider that pigeons met disperse between these two sites, depending on both the egg removal and limiting food resources strategies adopted in each site. In fact, several studies exist on the patterns of pigeon dispersal confirming that dispersal is a natural mechanism within a bird population (see, e.g. Boulinier et al. (2008); Greenwood and Harvey (1982); Hetmański (2007); Hinde (1952)). This dispersal which is classified between breeding and natal dispersal will be detailed later.

After introducing our model, we define our state constraint set which reflects the tradeoff between ecological considerations and urban citizen satisfaction. The viability theory of Aubin (1991) offers an interesting insight in this context. It provides theoretical concepts 
and practical tools to study the evolution of dynamical systems under state constraint. The main purpose of viability theory is to find a "viability domain", a subset of initial states such that there exists at least one evolution "viable" in the state constraint set, in the sense that at each time, the state of the evolution can be maintained inside it. Viability theory has been successfully used to model socio-ecological problem and study their governance, as in Béné et al. (2001), Bernard and Martin (2013) or Lara and Martinet (2009) (see Aubin et al. (2011) for others references). We propose here to formulate the conflicting viewpoints of urban citizen about pigeon population in the mathematical viability framework. We then study when and how it is possible to propose regulation satisfying both viewpoints. Using the viability algorithm developed in Falcone and Saint-Pierre (1987), we show the approximative viability kernel describing the possibility to control the density of an urban pigeon under social constraints.

The paper is organized as follows. Section 2 presents the model with a detailed description of all the parameters. Section 3 reformulates the management of the pigeon population as a viability problem and recalls some important definitions and theorems from the viability theory. Analytical and numerical results are given in Section 4. Section 5 discusses the ecological implications of the results of Section 4 in terms of the possibility of management of an urban pigeon population under social constraints, along with some perspectives.

\section{Model description}

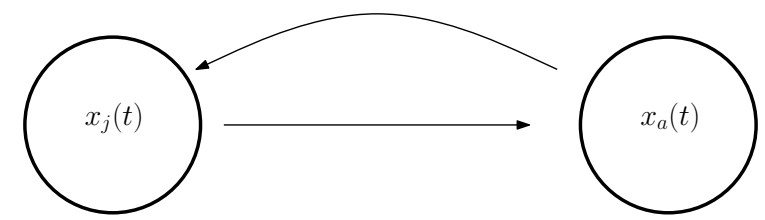

Figure 1: Life cycle of a pigeon population structured in two age classes

Before proceeding in the building of our model, we should give a description about the pigeon life cycle. During the first year the mortality of young pigeons is very high (see e.g. Haag-Wackernagel et al. (2006); Récapet et al. (2006)). they are fed infrequently by their parents and therefore are most susceptible to disease between the age of six to ten weeks. On the other hand, breeding age of pigeons is not uniform among the pigeon population. Normally, pigeons reach sexual maturity at about five to eight months. It is important therefore, when modeling the evolution of a pigeon population to consider an age-structured model. Thus, we consider that this population is devided into two age classes (see Figure 1): the juvenile pigeons and adult pigeons. Here, we consider that the juvenile pigeons class regroup both the young pigeons and the adult but non-reproductive pigeons. Of course, the mortality of young pigeons is more important of that of adult pigeons (As stressed in the beginning of this section), but here we suppose that the young and adult non-reproductive pigeons have the same mortality rate. The adult pigeons class is exclusive to the adult reproductive pigeons. Let us denote by $x_{j}(t)$ and $x_{a}(t)$ the size of juvenile and adult pigeons, 
respectively, at time $t$. Their dynamics, in an infinitesimal time $d t$, is given by

$$
\begin{aligned}
x_{j}(t+d t) & =x_{j}(t)+d t\left[n x_{a}(t)-m_{j} x_{j}(t)-p x_{j}(t)\right] \\
x_{a}(t+d t) & =x_{a}(t)+d t\left[-m_{a} x_{a}(t)+p x_{j}(t)\right]
\end{aligned}
$$

where $n$ and $m_{a}$ denote the reproduction and mortality rates of adult pigeons and $m_{j}$ the mortality rate of juvenile pigeon. The parameter $p$ denotes the transfer rate from juvenile to adult class.

As mentioned in the introduction, we assume that local authorities adopt both egg removal and limiting food resources strategies to control the pigeon population. By the following, we list the principal impacts of these management strategies on the pigeons ecology:

- egg removal may lead to an increase in laying frequency and the total yearly number of laid eggs,

- egg removal may lead to a decrease in the body conditions and egg quality of adult pigeons,

- the juvenile mortality is more important than that of adult mortality,

- the reproduction activity concerns only adult pigeons.

In order to incorporate these observations in the starting equations (1), we propose the following model ${ }^{1}$

$$
\begin{aligned}
& \dot{x}_{j}=n\left(x_{a}, r, s\right) x_{a}-m_{j}\left(x_{j}, r, s\right) x_{j}-p\left(x_{j}, r, s\right) x_{j} \\
& \dot{x}_{a}=-m_{a}\left(x_{a}, r, s\right) x_{a}+p\left(x_{j}, r, s\right) x_{j},
\end{aligned}
$$

where $r$ and $s$ denote, respectively, the egg removal and limiting food resources strategies. Knowing that these control strategies may differ from one site to another, our model must incorporate a spatial distribution of the pigeon population. Here, we are limited to two sub-populations of urban pigeons, having different rates of reproduction and mortality. Furthermore, knowing that

- egg removal and limiting food resources encourages the dispersal of pigeons, and starting from equation (2), we propose the following model ${ }^{2}$

$$
\begin{aligned}
& \dot{x}_{j i}=n_{i}\left(x_{a i}, r_{i}, s_{i}\right) x_{a i}-m_{j i}\left(x_{j i}, r_{i}, s_{i}\right) x_{j i}-p_{i}\left(x_{j i}, r_{i}, s_{i}\right) x_{j i}-\sum_{k=1}^{2}(-1)^{i+k} x_{j k} \phi_{j k}\left(x_{j k}, s_{k}\right) \\
& \dot{x}_{a i}=-m_{a i}\left(x_{a i}, r_{i}, s_{i}\right) x_{a i}+p_{i}\left(x_{j i}, r_{i}, s_{i}\right) x_{j i}-\sum_{k=1}^{2}(-1)^{i+k} x_{a k} \phi_{a k}\left(x_{a k}, r_{k}, s_{k}\right)
\end{aligned}
$$

\footnotetext{
${ }^{1}$ In order to lightening the presentation of this model, we omit the time dependency of $x_{a}$ and $x_{j}$.

${ }^{2}$ In order to lightening the presentation of this model, we omit the state dependency of the controls $r_{i}$ and $s_{i}$, for $i=1,2$.
} 
where $x_{j i}$ and $x_{a i}$ denote the size of juvenile and adult pigeon of population subject to removal strategy $r_{i}$ and food resource limitation strategy $s_{i}$, for $i=1,2$. The function $n_{i}(\cdot)$ describes the reproduction of adult pigeon; $m_{a i}(\cdot)$ and $m_{j i}(\cdot)$ describe the mortality of adult and juvenile pigeon, respectively, for $i=1,2$. The function $p_{i}(\cdot)$ represents the transfer rate from juvenile to adult. The functions $\phi_{j k}$ and $\phi_{a k}$ represents the dispersal rate of juvenile and adult pigeons from population $i$ to $k \neq i$, for $i=1,2$.

\subsection{Parameters description}

In this section, relying on the existing literature, we give a detailed description of the different parameters and functions which appeared in the equations of our model (3).

\subsubsection{Parameters description: without control actions}

Beside the sociological constraints, the annual survival and reproduction rates of any pigeon population depend certainly on many ecological factors which are specific to each city or country. Pigeons' ecology has not been much studied compared to others species, we have access to very few different estimations of demographic parameters. Here, we use the data reported by Murton et al. (1974), regarding feral pigeons breeding on Sandford Docks in Manchester (see Table 1). In that study, the survival rate of juveniles referred to the first sixth months of life. This survival rate should be considered density-dependent. In fact, in Kautz and Malecki (1991) the authors show that an increased survival of pigeons following an experimentally induced decrease in population density. In the absence of any external influence, the natural density-dependent mortality functions of a pigeon population $x_{i}$ can be taken as the following

$$
m\left(x_{i}\right)=\bar{m}_{i}\left(1+\frac{x_{i}}{M_{i}}\right)
$$

where the constant $\bar{m}_{i}$ defines the mortality rate of pigeon population $x_{i}$, and $M_{i}$ represents the carrying capacity of this population, for $i=1,2$.

Concerning the reproductive rate, expressed as the number of fledglings per female per year, we chose an approximative one from Murton et al. (1974) (see Table 1). The reproduction function should be considered density-dependent. In fact, reproduction of feral pigeons being high at low density and low at higher density Haag (1991); Kautz and Malecki (1991). In the absence of any external influence, the natural reproduction of the pigeon population $x_{i}$ can be taken as the following

$$
n\left(x_{i}\right)=\bar{n}_{i}\left(1-\frac{x_{i}}{M_{i}}\right),
$$

where the constant $\bar{n}_{i}$ defines the reproduction rate of the population $x_{i}$, for $i=1,2$.

The transfer rate is modeled here simply as a mortality. Concerning the dispersal function, we suppose that the dispersal decision of juvenile and adult pigeons depends on the size of local and neighboring pigeons according the following relations

$$
\phi_{a k}\left(x_{a k}\right)=\bar{\phi}_{a k} \frac{x_{j k}}{M_{k}+x_{a i}+x_{j i}} \quad \text { and } \quad \phi_{j k}\left(x_{j k}\right)=\bar{\phi}_{j k} \frac{x_{a k}}{M_{k}+x_{a i}+x_{j i}},
$$

where $\bar{\phi}_{a k}, \bar{\phi}_{j k} \in[0,1]$, for $k \neq i$ and $k, i \in\{1,2\}$.

Under managing actions, these functions should respect the different features listed at the 
beginning of this section. Especially, these functions should respect the fact that egg removal strategy increases the total yearly number of laid eggs and decrease the body condition of adult pigeons and their eggs' quality. We should also distinguish between adult pigeon and juvenile pigeon mortality. Furthermore, we should integrate the dispersal decision dependency on the egg removal and limiting food resources strategies. This dependency will be described in detail in Section 2.1.3.

\subsubsection{Control parameters description}

We suppose that the control parameters $r_{i}$ and $s_{i}$ may vary continuously between 0 and 1. The value $r_{i}=0\left(s_{i}=0\right.$, respectively) reflects the more tolerable removal strategy (food limitation strategy, respectively), and the value $r_{i}=1\left(s_{i}=1\right.$, respectively) reflects the more aggressive removal strategy (food limitation strategy, respectively) adopted in the ith site, for $i=1,2$. By the more tolerable strategy we mean simply the absence of any management strategy and by the more aggressive strategy the removing of all laid eggs or the elimination of all food resources.

\subsubsection{Parameters description: with control actions}

Under these management strategies, the mortality and reproduction functions should respect the following conditions:

$$
\left\{\begin{array}{l}
n_{i}\left(x_{i}, 1, s_{i}\right)=0 \\
n_{i}\left(x_{i}, 0,0\right)=n\left(x_{i}\right) \\
n_{i}\left(x_{i}, r_{i}, s_{i}\right) \leq n\left(x_{i}\right), \quad \text { for } \quad i=1,2
\end{array}\right.
$$

Observe that, without any external influence, the reproduction function is equal to the natural reproduction (given by equation (5)). In addition, this function should increase with respect to $r_{i}$ to reach a maximum reproduction rate (this in accordance with what is mentioned before). Furthermore, this reproduction function should decrease to reach zero when the eggs are totally removed. Concerning the mortality function, the following conditions should be satisfied

$$
\left\{\begin{array}{l}
m_{a i}\left(x_{i}, r_{i}, s_{i}\right) \geq m\left(x_{i}\right) \\
m_{a i}\left(x_{i}, 0,0\right)=m\left(x_{i}\right) \\
m_{j i}\left(x_{i}, r_{i}, s_{i}\right)=\nu m_{a i}\left(x_{i}, r_{i}, s_{i}\right), \quad \text { for } \quad i=1,2
\end{array}\right.
$$

with $\nu>1$. Also here, we observe that, without any external influence, the adult mortality function is identically equal to the natural mortality (given by equation (4)). In addition, this function should increase when removal eggs and limiting resources strategies are adopted. The introduced parameter $\nu>1$ reflects the fact that the mortality is more important among juvenile than that of adult pigeon. 
Similarly, as mentioned in the introduction, the pigeons dispersal is closely related to the local management strategies. In particular, this dispersal function depends on two crucial facts: the local level of breeding success and the food resources disposition. In fact, as observed in several studies (see, e.g. Greenwood and Harvey (1982); Harvey et al. (1997)), a wide range of bird species move to a new breeding locality following a poor or unsuccessful breeding attempt the previous year. A study conducted by Boulinier et al. (2008) on the breeding dispersal of kittiwakes, shows that individuals that lost their eggs on successful patches would attend their nest and come back to it the year after at a higher rate than individuals that lost their eggs on patches where their neighbors were also in failure. This suggests that an egg removal strategy can play an important role regarding the pigeons decisions relative to breeding site selection. Obviously, the dispersal activity is also related to the food resources availability. In fact, as reported in Hinde (1952), it is advantageous for all nomadic species (the great tit in this case) to disperse following a complete failure in the food supply. In addition we should differentiate between juvenile and adult dispersal. In fact, as mentioned in Greenwood and Harvey (1982), the dispersal is often strongly biased towards juveniles, relatively to adults which may also move from one breeding site to another but with some increasing (with age) tendency for philopatry (see e.g. Hetmański (2007)). According to these observations, the dispersal functions should respect the following conditions:

$$
\left\{\begin{array}{l}
\phi_{a i}\left(x_{a i}, 0,0\right)=\phi_{a i}\left(x_{a i}\right), \quad \phi_{j i}\left(x_{j i}, 0\right)=\phi_{j i}\left(x_{j i}\right), \\
\phi_{a i}\left(x_{a i}, r_{i}, s_{i}\right) \geq \phi_{a i}\left(x_{a i}\right), \quad \phi_{j i}\left(x_{j i}, s_{i}\right) \geq \phi_{j i}\left(x_{j i}\right), \quad \text { for } \quad i=1,2 .
\end{array}\right.
$$

\section{Material and methods}

In this section, we propose to use the mathematical viability theory to formulate the issue of the management of the pigeon population.

\subsection{The viability theory approach}

As mentioned in the introduction, the viability theory of Aubin (1991) can be used to study the behavior of dynamical systems under state space constraints. Let $X \subset \mathbb{R}^{n}$, for $n \geq 1$, be the state space of the system. We assume that the evolution of the system state $x(\cdot):[0,+\infty) \ni t \mapsto x(t) \in X$ depends on a external control action $u(\cdot) \in U(x(\cdot)) \subset \mathbb{R}^{p}$, for $p \geq 1$. Let $f: \mathbb{R}^{n} \times \mathbb{R}^{p} \rightarrow \mathbb{R}^{m}, m \geq 1$, be the function that defines the state evolution. This control dynamical system can be written as the following

$$
\begin{aligned}
& \dot{x}(t)=f(x(t), u(t)) \\
& u(t) \in U(x(t)) .
\end{aligned}
$$

A solution for this system is a trajectory $t \mapsto x(t)$ so that a measurable control function $t \mapsto u(t)$ exists and that the condition $u(t) \in U(x(t))$ is satisfied for almost every $t \geq 0$.

Viability constraints are described by a closed subset $K \subset X$ of the state space. They describe the viability of the system since the state of the system is no longer viable outside of $K$. We recall the definitions of the contingent cone and the viability domain. 
Definition 1. (Viable trajectory). Let $X$ be a finite dimensional vector space and let $K$ be a subset of $X$. A function $x(\cdot)$ from $[0,+\infty)$ to $X$ is viable in $K$ if

$$
\forall t \geq 0, x(t) \in K \text {. }
$$

Definition 2. (Viability). Let $X$ be a finite dimensional vector space and let $K$ be a subset of $X$. We say that $K$ is viable under system (10) if for any initial state $x_{0}$ of $K$, there exist a solution of (10) starting at $x_{0}$ which is viable in $K$.

Definition 3. (Contingent cone). Let $X$ be a finite dimensional vector space, $K$ be a subset of $X$ and $x \in K$. The contingent cone $T_{K}(x)$ to $K$ at $x$ is the set of all vectors $v \in X$ such that if there exist a sequence of $h_{n}>0$ converging to $0^{+}$and a sequence of $v_{n} \in X$ converging to $v$ such that

$$
\forall n \geq 0, x+h_{n} v_{n} \in K .
$$

Definition 4. (Viability domain). Let $X$ be a finite dimensional vector space and let $K$ be a subset of $X$. We say that $K$ is a viability domain of the controlled system (10) if and only if

$$
\forall x \in K, \quad F(x) \cap T_{K}(x) \neq \emptyset,
$$

where $F(x):=\{f(x, u)\}_{u \in U(x)}$.

Definition 5. (Viability kernel). The largest viability domain inside the state constraint set $K$ is called the viability kernel of the controlled system (10).

The link between viability domains and the existence of viable solutions is given by the following theorem.

Theorem 1. Consider a Marchaud ${ }^{3}$ control system $(U, f)$ and a closed subset $K \subset \operatorname{Dom}(U)$ of $X . K$ is viable under system (10) if and only if $K$ is a viability domain of (10).

Consequently, if the whole set $K$ is a viability domain, whatever be the initial state, there exists a control function that governs an evolution which remains in this set of state constraint. If state constraint set is not a viability domain, finding a subset which is the viability domain guarantees the existence of a viable evolution from any starting point inside.

\footnotetext{
${ }^{3}$ If satisfies the following conditions:

1. $\operatorname{Graph}(F):=\{(x, y) \in X \times X \mid y \in F(x)\}$ and $\operatorname{Dom}(F):=\{x \in X$ such that $F(x) \neq \emptyset\}$ are closed

2. the values of $F$ are convex

3. the growth of $F$ is sublinear, i.e. $\exists c>0$ such that $\forall x \in \operatorname{Dom}(F), \sup _{y \in F(x)}\|y\| \leq c(\|x\|+1)$.
} 


\subsection{Management of the pigeon population as a viability problem}

To reformulate, starting from system (3), the managing problem of pigeons as a viability problem, we first define our state constraint set. The state space consists of four variables (two in the case of one pigeon population): the adults and juveniles pigeons from each site. The constraint set is a subset of this four (or two respectively) dimensional space. As mentioned in the introduction, to achieve citizen satisfaction, the local authorities fix an upper limit on the total number of the pigeon population. Once this population exceeds this upper limit, the local authorities try to reduce this increased pigeon population by using different managing methods. Since this satisfaction may be different between sites, this upper limit may be different from one site to another. On the other hand, urban citizens are also supposed to be dissatisfied when the pigeon welfare is not ensured. Welfare could be represented by several state variables, for example the mean health state, or the lifetime. As a first approach, in order to keep a low number of state variables for computational reasons, we consider that the pigeon welfare can also be represented by the level of the total population: a low number of pigeons is a proxy a bad welfare conditions for the considered pigeon population. The threshold values of this constraint set reflects the trade-off between ecological considerations and urban citizen satisfaction. The constraint set can be then given by the following

$$
(K) \begin{cases}\alpha_{i} M_{i} \leq x_{j i}(t)+x_{a i}(t) \leq \beta_{i} M_{i}, & \forall t \geq 0, \\ x_{a i}(t) \geq 0, & \forall t \geq 0, \\ x_{j i}(t) \geq 0, & \forall t \geq 0,\end{cases}
$$

where $\alpha_{i}, \beta_{i} \in[0,1]$ determine the lower and upper limits, respectively, for $i \in\{1,2\}$. Finally, the viability problem that we have to solve is the following one

$$
\left\{\begin{array}{l}
\dot{x}_{j i}=n_{i}\left(x_{a i}, r_{i}, s_{i}\right) x_{a i}-m_{j i}\left(x_{j i}, r_{i}, s_{i}\right) x_{j i}-p_{i}\left(x_{j i}, r_{i}, s_{i}\right) x_{j i}-\sum_{k=1}^{2}(-1)^{i+k} x_{j k} \phi_{j k}\left(x_{j k}, r_{k}, s_{k}\right) \\
\dot{x}_{a i}=-m_{a i}\left(x_{a i}, r_{i}, s_{i}\right) x_{a i}+p_{i}\left(x_{j i}, r_{i}, s_{i}\right) x_{j i}-\sum_{k=1}^{2}(-1)^{i+k} x_{a k} \phi_{a k}\left(x_{a k}, r_{k}, s_{k}\right) \\
\alpha_{i} M_{i} \leq x_{j i}(t)+x_{a i}(t) \leq \beta_{i} M_{i}, \quad \forall t \geq 0, \\
x_{a i}(t), x_{j i}(t) \geq 0, \quad \forall t \geq 0, \\
r_{i}, s_{i} \in[0,1], \quad \forall i \in\{1,2\} .
\end{array}\right.
$$

\subsection{Algorithm for the approximation of the viability kernel}

The computation of the viability kernel for the pigeon problem is done with the $k d$-tree viability framework from Alvarez et al. (2013) and Rouquier et al. (2015). It is available on-line (more information from Alvarez et al. (2016)). It produces a partition of the search

space into leaves of a $k d$-tree. With each viable leaf comes a control vector for which the 
test point associated with the leaf stays in the approximation of the viability kernel for the specific control vector. This information is generally only used to control trajectories near the boundary of the viability kernel, since inside the viability kernel, when the time step is small enough, every control is viable. Control information allows to understand what happens near the boundary of the viability kernel. It also allows to develop action plans to control viable trajectories, or to find trajectories which optimizes some cost function while maintaining the system in the viability kernel. In the $k d$-tree viability framework it is possible to order the discretized controls before they are tested. The resulting approximation of the viability kernel is not modified, but registered controls depend on the chosen order. For example, food control can be tested before any egg removal strategy when it is easier to implement in the field. More general strategies can be tested, for example careful strategies which stay far from the boundary like in Alvarez and Martin (2011).

The $k d$-tree viability framework provides also for two and tree dimensional problems a description of the viability kernel in VTK format, in order to allow vizualisation facilities with Paraview (AHRENS et al. (2005)).

\section{Results}

This section presents the main contribution of this paper. We analyze, in two different cases, the viability problem given by system (14).

\subsection{Case of one age-structured population}

Let us denote by $\partial K$ the boundary of the set of state constraint $K$. In this case, we have $\partial K=K_{1} \cup K_{2} \cup K_{3} \cup K_{4}$, where

$$
\begin{aligned}
& K_{1}=\left\{\left(x_{j}, x_{a}\right) \in \mathbb{R}^{2} \mid x_{a}=0 \text { and } \alpha M<x_{j}<\beta M\right\} \\
& K_{2}=\left\{\left(x_{j}, x_{a}\right) \in \mathbb{R}^{2} \mid x_{a}+x_{j}=\alpha M\right\} \\
& K_{3}=\left\{\left(x_{j}, x_{a}\right) \in \mathbb{R}^{2} \mid \alpha M<x_{a}<M \text { and } x_{j}=0\right\} \\
& K_{4}=\left\{\left(x_{j}, x_{a}\right) \in \mathbb{R}^{2} \mid x_{a}+x_{j}=\beta M\right\}
\end{aligned}
$$

In order to have an idea on the viability kernel in this case, we consider the following natality, mortality and transfer functions

$$
\left\{\begin{array}{l}
n\left(x_{a}, r, s\right)=(1-r)\left(1-s^{2}\right) n\left(x_{a}\right) \\
m_{a}\left(x_{a}, r, s\right)=(1+r)\left(1+s^{2}\right) m\left(x_{a}\right) \\
m_{j}\left(x_{j}, r, s\right)=\nu m\left(x_{j}, r, s\right) \\
p\left(x_{j}, r, s\right)=\rho m_{j}\left(x_{j}, r, s\right)
\end{array}\right.
$$

where $n(\cdot)$ and $m(\cdot)$ are given by equations (4)-(5), $r, s \in[0,1], \nu>1$ and $\rho<1$. Remark that these functions are in concordance with the conditions given by equations (7)-(8). The use of the square of $s$ (instead of $s$ ) is in order to weaken the impact of food resources management compared to that of egg removal strategies. 


\subsubsection{Analytical results}

Before the numerical computation of the viability kernel, it is necessary to have an idea on the equilibria of system (2). In fact, depending on the control parameters $r$ and $s$, the equilibria of (2) may be inside or outside the constraint set $K$ (see Figure (2)). Then, it is necessary two have an idea on how these controls modify the equilibria of this system. This is given by the following proposition. Firstly, we define, for each pair $r, s \in[0,1]$, the following quantity

$$
\theta=\frac{\rho \bar{n}(1-r)\left(1-s^{2}\right)-(1+\rho) \bar{m}(1+r)\left(1+s^{2}\right)}{\rho \bar{n}(1-r)\left(1-s^{2}\right)+(1+\rho) \bar{m}(1+r)\left(1+s^{2}\right)} .
$$

We have the following proposition.

Proposition 1. Consider system (2) and let $K$ be the set of state constraint given by equation (13). If $\theta \geq 0$, where $\theta$ is given by equation (17), then system (2) admits a unique equilibrium $\left(x_{j}^{\star}, x_{a}^{\star}\right)$ in the positive orthant, where

$$
\begin{aligned}
x_{j}^{\star} & =(-1 / 2+\sqrt{1 / 4+\theta(1+\theta) / \nu \rho}) M, \\
x_{a}^{\star} & =\theta M .
\end{aligned}
$$

Furthermore, this positive equilibrium belong to $K$ if and only if

$$
\alpha \leq \theta-1 / 2+\sqrt{1 / 4+\theta(1+\theta) / \nu \rho} \leq \beta .
$$

proof. In this case, the model of the one age-structured pigeon population is given by

$$
\begin{aligned}
& \dot{x}_{j}=(1-r)\left(1-s^{2}\right) n\left(x_{a}\right) x_{a}-\nu(1+\rho)(1+r)\left(1+s^{2}\right) m\left(x_{j}\right) x_{j} \\
& \dot{x}_{a}=-(1+r)\left(1+s^{2}\right) m\left(x_{a}\right) x_{a}+\nu \rho(1+r)\left(1+s^{2}\right) m\left(x_{j}\right) x_{j} .
\end{aligned}
$$

At the equilibrium, we have

$$
\begin{aligned}
(1-r)\left(1-s^{2}\right) n\left(x_{a}\right) x_{a} & =\nu(1+\rho)(1+r)\left(1+s^{2}\right) m\left(x_{j}\right) x_{j} \\
m\left(x_{a}\right) x_{a} & =\nu \rho m\left(x_{j}\right) x_{j} .
\end{aligned}
$$

Then, from equation (21), we have

$$
\begin{aligned}
\frac{(1-r)\left(1-s^{2}\right)}{(1+r)\left(1+s^{2}\right)} n\left(x_{a}\right) x_{a} & =\nu(1+\rho) m\left(x_{j}\right) x_{j} \\
m\left(x_{a}\right) x_{a} & =\nu \rho m\left(x_{j}\right) x_{j} .
\end{aligned}
$$

Combining these two latter equations gives,

$$
\underbrace{\frac{(1-r)\left(1-s^{2}\right)}{(1+r)\left(1+s^{2}\right)} n\left(x_{a}\right) x_{a}}_{\psi_{1}\left(x_{a}\right)}=\underbrace{\frac{(1+\rho)}{\rho} m\left(x_{a}\right) x_{a}}_{\psi_{2}\left(x_{a}\right)} .
$$


Remark, from the definition of $n(\cdot)$ and $m(\cdot)$ (given by equations (4)-(5)), that the function $\psi_{1}$ is positive over $[0, M], \psi_{1}(0)=\psi_{1}(M)=0$, and that $\psi_{1}$ is increasing over $[0, M / 2]$ and decreasing over $[M / 2, M]$ and that is a concave function which is always under the straight line of slope $\psi_{1}^{\prime}(0)=\frac{(1-r)\left(1-s^{2}\right)}{(1+r)\left(1+s^{2}\right)} \bar{n}$. Moreover, the function $\psi_{2}$ is a convex increasing function such that $\psi_{2}(0)=0$ and that $\psi_{2}$ is always upper the strait line of slope $\psi_{2}^{\prime}(0)=\frac{(1+\rho)}{\rho} \bar{m}$. Consequently, equation (23) has at least one trivial solution $x_{a}=0$ and one non-trivial which correspond to the case when $\psi_{1}^{\prime}(0)>\psi_{2}^{\prime}(0)$, i.e. when

$$
\rho \bar{n}(1-r)\left(1-s^{2}\right) \geq(1+\rho) \bar{m}(1+r)\left(1+s^{2}\right) .
$$

this non-trivial solution is given by $x_{a}^{\star}=\frac{\rho \bar{n}(1-r)\left(1-s^{2}\right)-(1+\rho) \bar{m}(1+r)\left(1+s^{2}\right)}{\rho \bar{n}(1-r)\left(1-s^{2}\right)+(1+\rho) \bar{m}(1+r)\left(1+s^{2}\right)} M$. Thus, from (22), the non-trivial equilibrium point is given by the following

$$
\begin{aligned}
x_{j}^{\star} & =\frac{-1+\sqrt{1+\frac{4}{\nu \rho}(1+\theta) \theta}}{2} M, \\
x_{a}^{\star} & =\theta M,
\end{aligned}
$$

where $\theta=\theta(r, s)$ is given by equation (17). It is clear that this equilibrium point belongs to the constraint set $K$ if and only if equation (19) holds.

By the following proposition, we show that, for each fixed $r, s \in[0,1]$, the non-trivial equilibrium (when it exists) is locally exponentially stable. In other words, there exists a neighborhood of $\left(x_{j}^{\star}, x_{a}^{\star}\right)$ such that from any starting point the trajectory remains in this neighborhood and converges exponentially to $\left(x_{j}^{\star}, x_{a}^{\star}\right)$.

Proposition 2. Consider system (2). The unique non-trivial equilibrium point $\left(x_{j}^{\star}, x_{a}^{\star}\right)$, when it exists, is locally exponentially stable.

proof. The Jacobian matrix of dynamics (2) at the equilibrium $x^{\star}=\left(x_{j}^{\star}, x_{a}^{\star}\right)$ is given by:

$$
J\left(x^{\star}\right):=\left(\begin{array}{cc}
-\nu(1+\rho) A & B \\
\nu \rho A & -C
\end{array}\right),
$$

where

$$
\begin{gathered}
A=(1+r)\left(1+s^{2}\right) \bar{m}\left(1+\frac{2}{M} x_{j}^{\star}\right) \quad \text { and } \quad B=(1-r)\left(1-s^{2}\right) \bar{n}\left(1-\frac{2}{M} x_{a}^{\star}\right) . \\
C=(1+r)\left(1+s^{2}\right) \bar{m}\left(1+\frac{2}{M} x_{a}^{\star}\right) .
\end{gathered}
$$

Let $\lambda_{1}$ and $\lambda_{2}$ be the eigenvalues of $J\left(x^{\star}\right)$. Knowing that $J\left(x^{\star}\right)$ is of dimension two, then we have the following property

$$
\lambda_{1}+\lambda_{2}=-\nu(1+\rho) A-C \quad \text { and } \quad \lambda_{1} \lambda_{2}=\nu(1+\rho) A C-\nu \rho A B .
$$




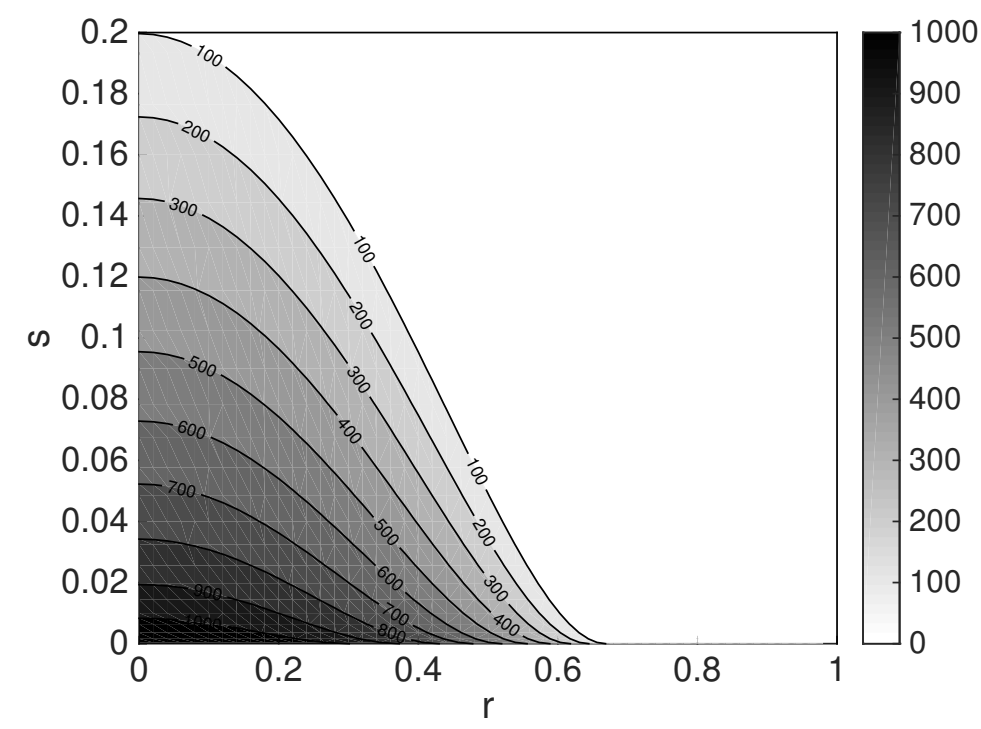

Figure 2: Influence of the values of $r$ and $s$ on the equilibrium point $\left(x_{j}^{\star}, x_{a}^{\star}\right)$ of system (2). The total population at the equilibrium $x_{j}^{\star}+x_{a}^{\star}$ is compared to the carrying capacity, for each $r, s \in[0,1]$. All the parameter values used for this simulation are given in Table 1.

Then, the Jacobian matrix $J\left(x^{\star}\right)$ is Hurwitz, i.e having eigenvalues with negative real parts, if and only if $\lambda_{1}+\lambda_{2}<0$ and $\lambda_{1} \lambda_{2}>0$. It is clear that $\lambda_{1}+\lambda_{2}<0$. Let then check the sign of $\lambda_{1} \lambda_{2}$. We have

$$
\begin{aligned}
\lambda_{1} \lambda_{2} & =\nu A[\nu(1+\rho) C-\rho B] \\
& =\nu A\left[(1+\rho)(1+r)\left(1+s^{2}\right) \bar{m}\left(1+\frac{2}{M} x_{a}^{\star}\right)-\rho(1-r)\left(1-s^{2}\right) \bar{n}\left(1-\frac{2}{M} x_{a}^{\star}\right)\right] \\
& =\nu A\left[(1+\rho)(1+r)\left(1+s^{2}\right) \bar{m}(1+2 \theta)-\rho(1-r)\left(1-s^{2}\right) \bar{n}(1-2 \theta)\right] \\
& =\nu A\left[\rho(1-r)\left(1-s^{2}\right) \bar{n}+(1+\rho)(1+r)\left(1+s^{2}\right) \bar{m}\right] \theta .
\end{aligned}
$$

Knowing, from Proposition (1), that the existence of non-trivial positive equilibrium $x^{\star}$ holds if and only if $\theta>0$, then we have $\lambda_{1} \lambda_{2}>0$. Hence, the Jacobian matrix $J\left(x^{\star}\right)$ given in (25) is Hurwitz, which establishes that $x^{\star}$ is locally exponentially stable for (2).

Thanks to Proposition 1 and Proposition 2, we know that the viability kernel of system (2) is non-empty; it contains at least the equilibrium point $x^{\star}$ and a neighborhood of $x^{\star}$. Even if the equilibrium point is inside the set of state constraint $K$, for some initial condition, a trajectory of system (2) may violate the boundary of $K$ (see for example Figure 3 ). This show the importance of dealing with an adaptive control instead of fixed ones. And the question is to determine the greatest set of initial conditions $V \subset K$ such that for each $x_{0} \in V$ the evolution of the population stay in $V$ for a given $r$ and $s$. Before that, we examine if the viability conditions are satisfied on the boundaries of $K$. This is given by the following proposition. 

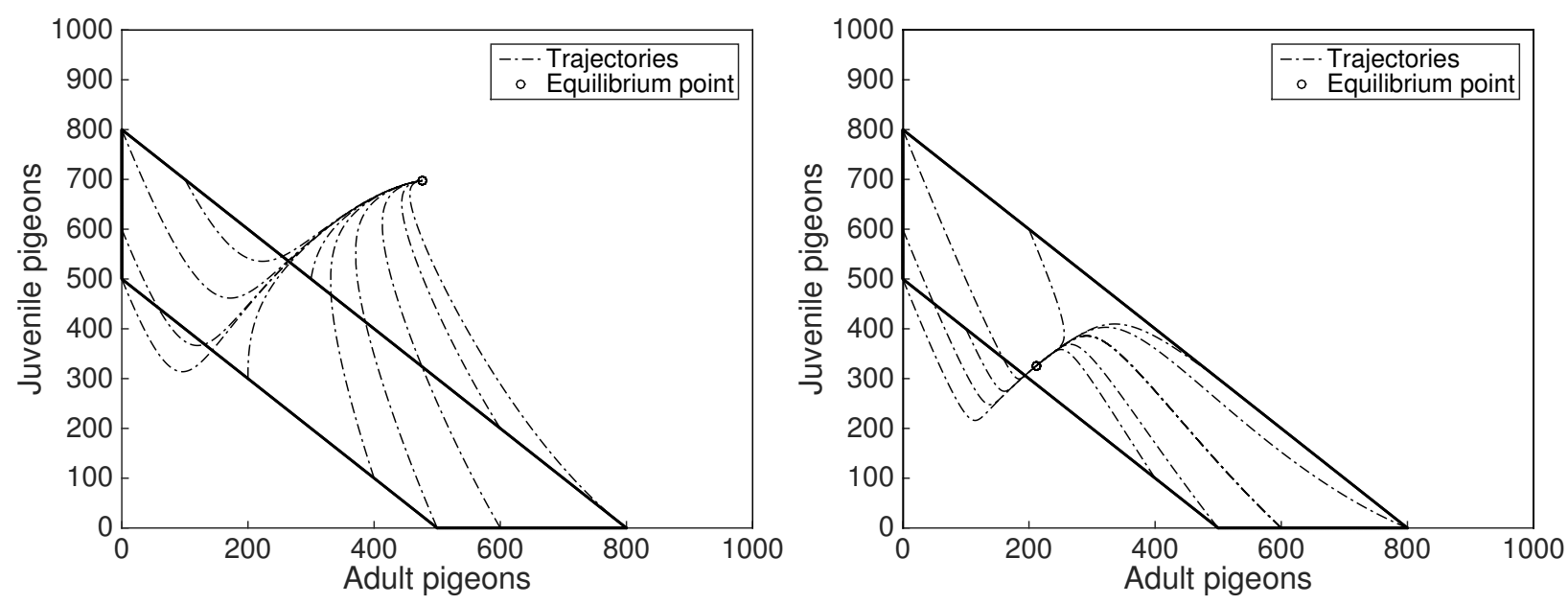

Figure 3: Phase portrait of system (2) for $s=0, r=0$ (left) and $s=0.1, r=0.2$ (right). All the parameter values used for these simulations are given in Table 1.

Let us introduce the following quantities

$$
S=\frac{h(r, s) \bar{n}+(2 \nu \beta+\nu-1) \bar{m}}{h(r, s) \bar{n}+(1+\nu) \bar{m}} M, \quad P=\frac{\nu \beta(1+\beta) \bar{m}}{h(r, s) \bar{n}+(1+\nu) \bar{m}} M^{2}
$$

where $h(r, s)$ is defined by

$$
h(r, s)=\frac{(1-r)\left(1-s^{2}\right)}{(1+r)\left(1+s^{2}\right)} .
$$

Proposition 3. Consider system (2). Let $K$ be the set of state constraint given by equation (13) with boundary $\partial K=K_{1} \cup K_{2} \cup K_{3} \cup K_{4}$. The viability condition is satisfied ${ }^{4}$ on $K_{1} \cup K_{3}$ and it is not satisfied on $K_{2}$. Concerning the boundary $K_{4}$, we distinguish two different cases:

- if $S^{2}-4 P \leq 0$ then the viability condition is satisfied on $K_{4}$,

- if $S^{2}-4 P>0$ we distinguish two different sub-cases:

- if for every $x_{a} \in[0, \beta M]$ there exist $r, s \in[0,1]$ such that $x_{a} \in\left[0, \hat{x}_{a}\right] \cup\left[\check{x}_{a}, \beta M\right]$ then the viability condition is satisfied on $K_{4}$,

- if for every $x_{a} \in[0, \beta M]$ and every $r, s \in[0,1]$ we have $x_{a} \in\left[\hat{x}_{a}, \check{x}_{a}\right]$ then the viability condition is not satisfied on $K_{4}$,

where $S$ and $P$ are given by equation (29), $\hat{x}_{a}=\frac{S-\sqrt{S^{2}-4 P}}{2}$ and $\check{x}_{a}=\frac{S+\sqrt{S^{2}-4 P}}{2}$.

\footnotetext{
${ }^{4}$ We say that the viability condition is satisfied on a boundary of $K$, if starting from this boundary there is always a control for which the trajectory of system (2) pointwises inside $K$.
} 
proof. Let $\left(x_{j}, x_{a}\right) \in \partial K$. If $\left(x_{j}, x_{a}\right)$ belongs to $K_{1}$, then $T_{K}\left(x_{j}, x_{a}\right)=\mathbb{R} \times \mathbb{R}^{+}$and, straightforwardly from equation (20), we have $\dot{x}_{a} \geq 0$ and $\dot{x}_{j} \leq 0$, then the viability condition, $T_{K}\left(x_{j}, x_{a}\right) \cap F\left(x_{j}, x_{a}\right) \neq \emptyset$, is satisfied on $K_{1}$. Similarly, if $\left(x_{j}, x_{a}\right)$ belongs to $K_{3}$, then $T_{K}\left(x_{j}, x_{a}\right)=\mathbb{R}^{+} \times \mathbb{R}$, and in this case we have $\dot{x}_{a} \leq 0$ and $\dot{x}_{j} \geq 0$, then the viability condition is satisfied on $K_{3}$. If $\left(x_{j}, x_{a}\right)$ belongs to $K_{2} \backslash\{(\alpha M, 0),(0, \alpha M)\}$, then $T_{K}\left(x_{j}, x_{a}\right)=\left\{(u, v) \in \mathbb{R}^{2}: u+v \geq 0\right\}$, and the viability condition is satisfied on $K_{2} \backslash\{(\alpha M, 0),(0, \alpha M)\}$ if and only if $\dot{x}_{a}+\dot{x}_{j} \geq 0$. Similarly, If $\left(x_{a}, x_{j}\right)$ belongs to $K_{4} \backslash\{(\beta M, 0),(0, \beta M)\}$, then $T_{K}\left(x_{j}, x_{a}\right)=\left\{(u, v) \in \mathbb{R}^{2}: u+v \leq 0\right\}$, and the viability condition is satisfied on $K_{4} \backslash\{(\beta M, 0),(0, \beta M)\}$ if and only if $\dot{x}_{a}+\dot{x}_{j} \leq 0$. Remark that

$$
\dot{x}_{a}+\dot{x}_{j}=\underbrace{\left(n\left(x_{a}, r, s\right)-m_{a}\left(x_{a}, r, s\right)\right) x_{a}-m_{j}\left(x_{j}, r, s\right) x_{j}}_{\Delta\left(x_{j}, x_{a}\right)} .
$$

The sign of $\Delta$ determines if the viability condition is satisfied or not on the boundaries $K_{2} \backslash$ $\{(\alpha M, 0),(0, \alpha M)\}$ and $K_{4} \backslash\{(\beta M, 0),(0, \beta M)\}$. Concerning $K_{2}$, we can straightforwardly conclude (for continuity reason), from the fact that $\Delta\left(x_{j}, 0\right)<0$ for every $x_{j}>0$, that the viability condition is not satisfied on $K_{2}$. Concerning $K_{4} \backslash\{(\beta M, 0),(0, \beta M)\}$, using the fact that on this boundary we have $x_{j}=\beta M-x_{a}$, we obtain that

$$
\operatorname{sign}\left(\Delta\left(x_{j}, x_{a}\right)\right)=-\operatorname{sign}\left(x_{a}^{2}-S x_{a}+P\right),
$$

where $S$ and $P$ are given by equation (29). By studying the solutions of the quadratic equation $\Delta\left(x_{j}, x_{a}\right)=0$, we can have a complete idea on the sign of $\Delta\left(x_{j}, x_{a}\right)$ for every $\left(x_{j}, x_{a}\right) \in K_{4} \backslash\{(\beta M, 0),(0, \beta M)\}$. Remark that on $(\beta M, 0)$ we have always $\dot{x}_{a} \geq 0$ and $\dot{x}_{a}+\dot{x}_{j} \leq 0$ then the viability condition is always satisfied in this point. Finally, remark that on $(0, \beta M)$ we have always $\dot{x}_{j} \geq 0$. Then the viability condition in $(0, \beta M)$ is only constrained by the sign of $\dot{x}_{a}+\dot{x}_{j}$ on $K_{4}$, which is already studied.

Remark 1. The fact that the viability condition is not satisfied on $K_{2}$ (or $K_{4}$ ) does not mean that is cannot be satisfied on a subset of $K_{2}$ (or $K_{4}$ ).

\subsubsection{Numerical computation of the viability kernel}

In this section, we compute numerically the viability kernel of problem (14), in four different cases: case $1(\alpha=0.3$ and $\beta=0.8)$, case $2(\alpha=0.5$ and $\beta=0.8)$, case $3(\alpha=0.5$ and $\beta=0.6)$ and case $4(\alpha=0.7$ and $\beta=0.8)$. Recall that $\alpha M$ and $\beta M$ represent, respectively, the lower and upper boundary of the constraint set $K$, where $M$ is the carrying capacity relative to this pigeon population. These viability kernels are represented by Figure 4 and Figure 5. The used parameter values are summarized by Table 1 . These viability kernels are accompanied by a color gradient on the boundaries of the state constraint set $K$. This color gradient indicates the control values needed for $r$ and $s$ to maintain the trajectories, starting near the boundaries, inside $K$. These control values are spread over different levels: "min", "low", "medium" and "high". The "min" value indicates the null control values. The "low" value indicates the non-null control values which are less than 0.3 . The "medium" value 


\begin{tabular}{|l|c|c|c|}
\hline Parameters & Values & Units & Reference \\
\hline $\bar{n}_{i}$ & 3 & 1/Year & Murton et al. (1974) \\
$\bar{m}_{i}$ & 0.34 & 1/Year & Murton et al. (1974) \\
$\bar{m}_{j}$ & 0.43 & 1/Year & Murton et al. (1974) \\
$M_{1}$ & 1000 & Number/Volume & Fictif \\
$M_{2}$ & 500 & Number/Volume & Fictif \\
$\nu$ & $\bar{m}_{j} / \bar{m}_{i}$ & - & - \\
$\rho$ & $1-0.43-0.1$ & - & - \\
\hline
\end{tabular}

Table 1: Table summarizing the used parameter values.

indicates the control values between 0.4 and 0.6 and the "high" value indicates the control values between 0.7 and 0.9 .

We clearly observe, from Figure 4 and Figure 5, that the common fact between these four cases is that the viability kernel is always non empty and that the constraint set $K$ is not a viability domain. This confirms the analytical result given by Proposition 2 , that the viability condition is not satisfied on the boundary $K_{2}$ and not always satisfied on the boundary $K_{4}$. Furthermore, we observe that the upper boundary $K_{4}$ is the only expensive one, where (in particular regions of this boundary) we should activate an egg removal or limiting food resources strategies to prevent that the pigeon population violates the constraint set. By Figure 4, we represent the viability kernel obtained by activating the limiting resource strategy at the first. In this case, we observe that a strong limiting resource strategy is needed in order to maintain the population inside the constraint set. By Figure 5, we represent the viability kernel obtained by activating the removal egg strategy at the first. In this case, we observe that a medium egg removal strategy is sufficient to maintain the population inside the constraint set. This can be simply justified from the fact that the egg removal impact is supposed more immediate than the food resources management (see (16)). Moreover, we can see that there is a level on juvenile and adult pigeons under which the population cannot be recovered.

\subsection{Case of two populations}

In this section, we analyze the role of the dispersal mechanism within a pigeon population and show the reliability to take into account such mechanism for the effectiveness of a management strategy. For this, we suppose that the pigeon population is splitted into two subpopulations. In order to simplify the representation of a viability kernel in this case, we suppose that the first population is structured as in system (2) but we suppose some homogeneity for the neighboring population where we don't differentiate between juvenile and adult pigeons. In addition, we suppose that this neighboring population is not subject to any management strategy. In fact, this neighboring population provides just a disturbing role for the first controlled population. The aim of this section is to provide a comparison with the previous one in order to show the dispersal role on the approximated viability kernel. For this, we chose the particular example corresponding to $700 \leq x_{j}+x_{a} \leq 800$ for which the viability kernel and the associated control are represented by Figure 5-(right-bottom). 

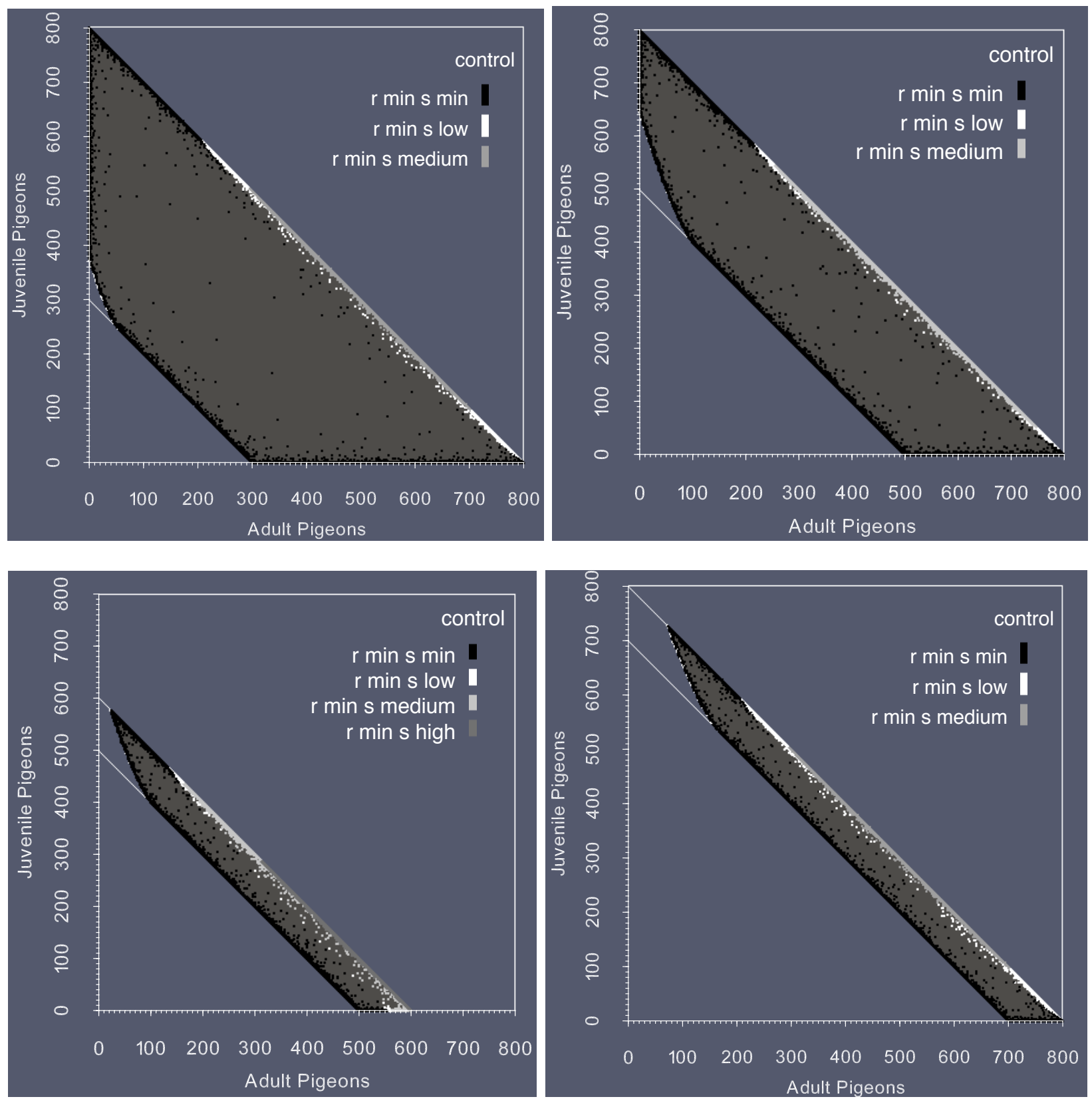

Figure 4: Viability kernel (in dark grey) for the viability problem 2. The constraint set is delimited by the white oblique lines and the axes. The points where the first control $(r=0, s=0)$ tested is viable appear in black. Points were $s$ is not minimum have a bigger size and colored light gray.

We consider the following system

$$
\begin{aligned}
& \dot{x}_{j}=n\left(x_{a}, r, s\right) x_{a}-m_{j}\left(x_{j}, r, s\right) x_{j}-p\left(x_{j}, r, s\right) x_{j}+\gamma \phi_{2}\left(x_{2}\right) x_{2}-\phi_{j}\left(x_{j}, s\right) x_{j} \\
& \dot{x}_{a}=-m_{a}\left(x_{a}, r, s\right) x_{a}+p\left(x_{j}, r, s\right) x_{j}+(1-\gamma) \phi_{2}\left(x_{2}\right) x_{2}-\phi_{a}\left(x_{a}, r, s\right) x_{a} \\
& \dot{x}_{2}=\left(n\left(x_{2}\right)-m_{a}\left(x_{2}\right)\right) x_{2}-\phi_{2}\left(x_{2}\right) x_{2}+\phi_{j}\left(x_{j}, s\right) x_{j}+\phi_{a}\left(x_{a}, r, s\right) x_{a}
\end{aligned}
$$



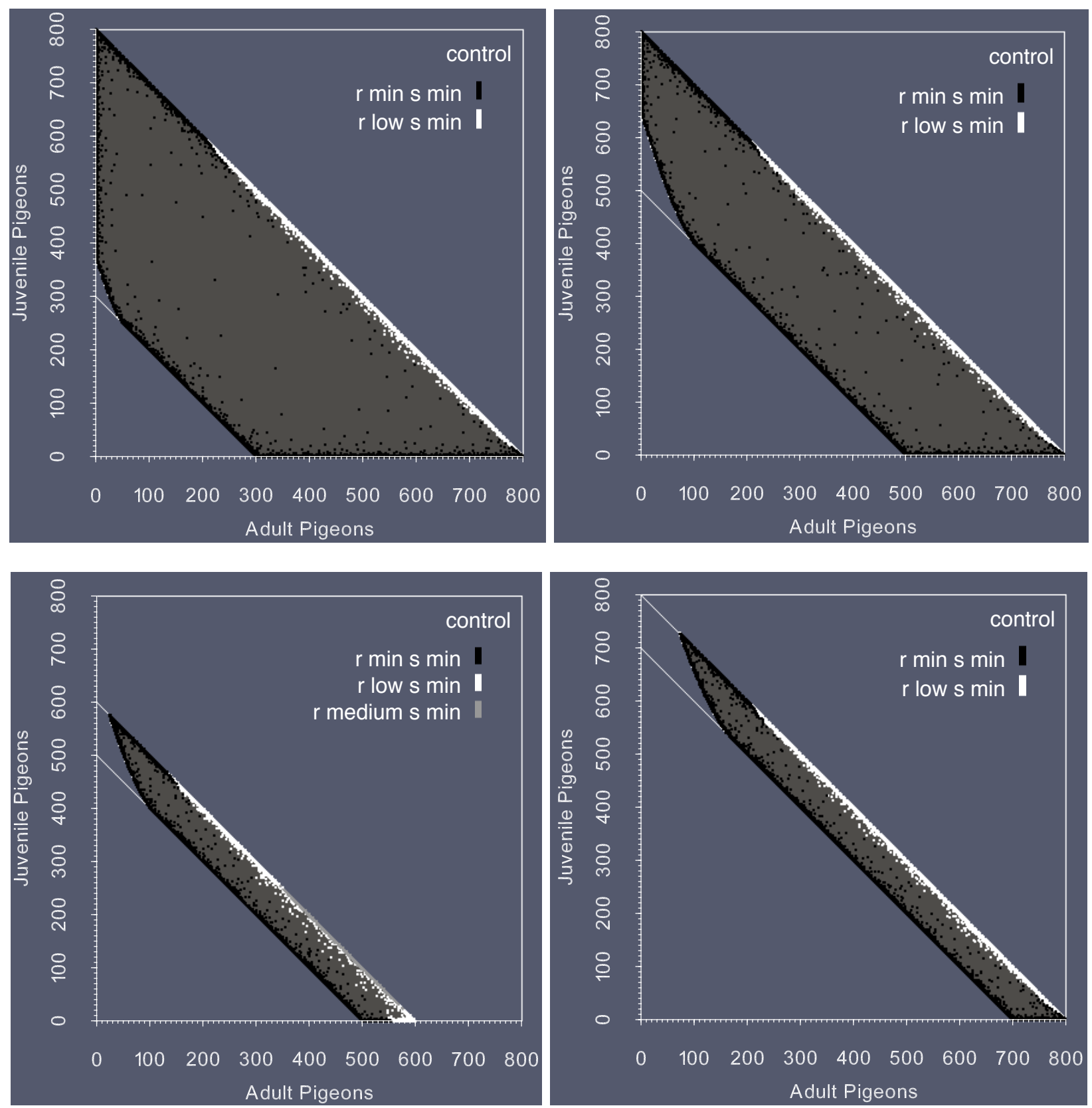

Figure 5: Viability kernel (in dark grey) for the viability problem 2. The constraint set is delimited by the white oblique lines and the axes. The points where the first control $(r=0, s=0)$ tested is viable appear in black. Points were $r$ is not minimum have a bigger size and colored light gray.

where $\gamma=4 / 7$ and $n, m_{a}, m_{j}, p$ have the same values as in previous section. Two examples of dispersal functions are proposed:

$$
\phi_{2}\left(x_{2}\right)=0.7, \quad \phi_{a}\left(x_{a}, r, s\right)=0.1, \quad \text { and } \quad \phi_{j}\left(x_{j}, r, s\right)=0.5 \text {, }
$$



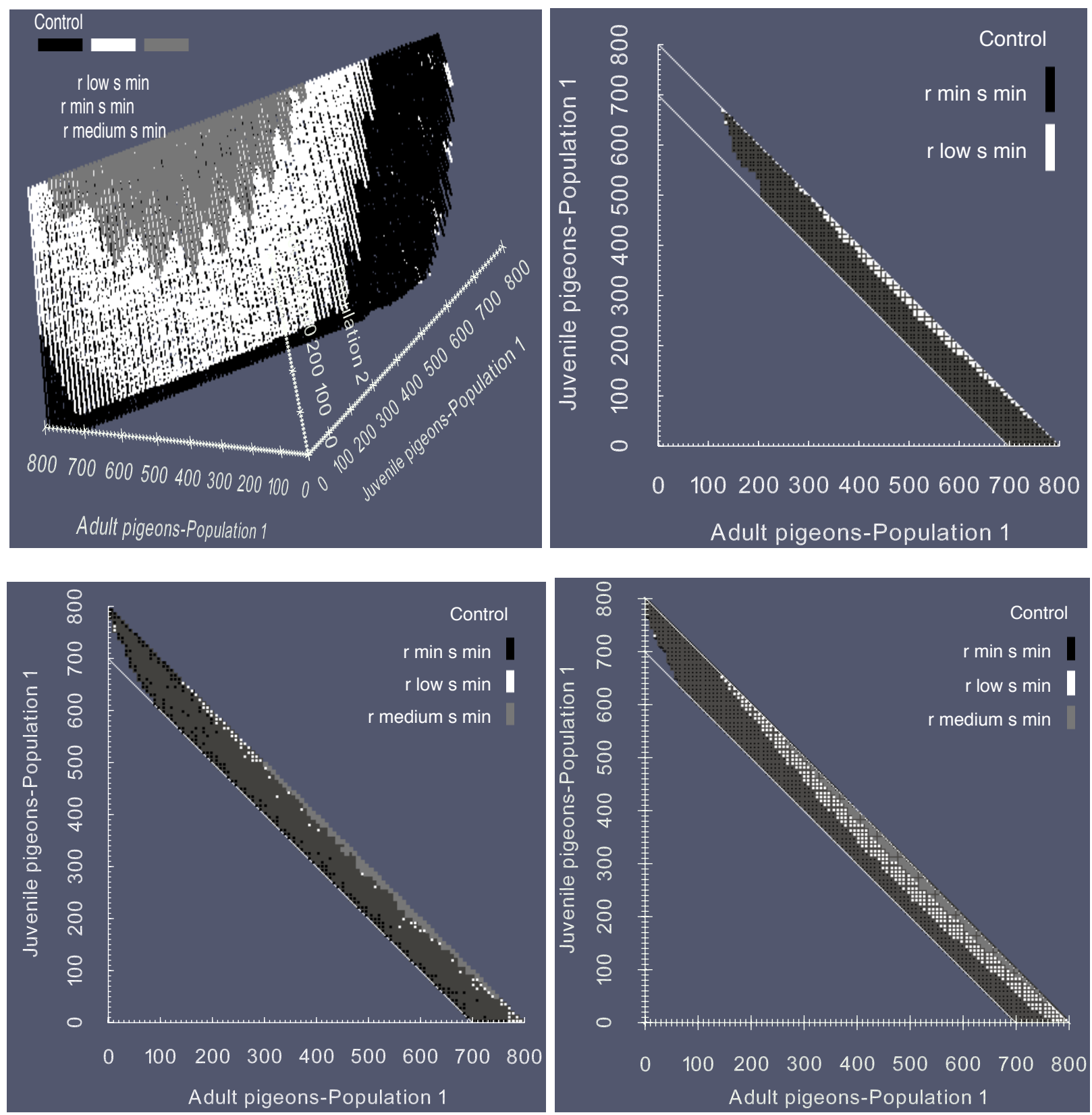

Figure 6: Figure left-top represents the viability kernel for the viability problem (32), in the case of the constant dispersal functions (33). Three clips at three different levels of the viability kernel: a first clip (right-top) for $x_{2}=0$, a second clip (left-bottom) for $x_{2}=450$ and the third clip (right-bottom) for $x_{2}=500$.

and

$\phi_{2}\left(x_{2}\right)=\frac{0.7 x_{2}}{M_{2}+x_{j}+x_{a}}, \quad \phi_{a}\left(x_{a}, r, s\right)=(1+r)(1+s) \frac{0.1 x_{j}}{M_{1}+x_{2}}, \quad \phi_{j}\left(x_{j}, s\right)=(1+s) \frac{0.5 x_{a}}{M_{1}+x_{2}}$. 

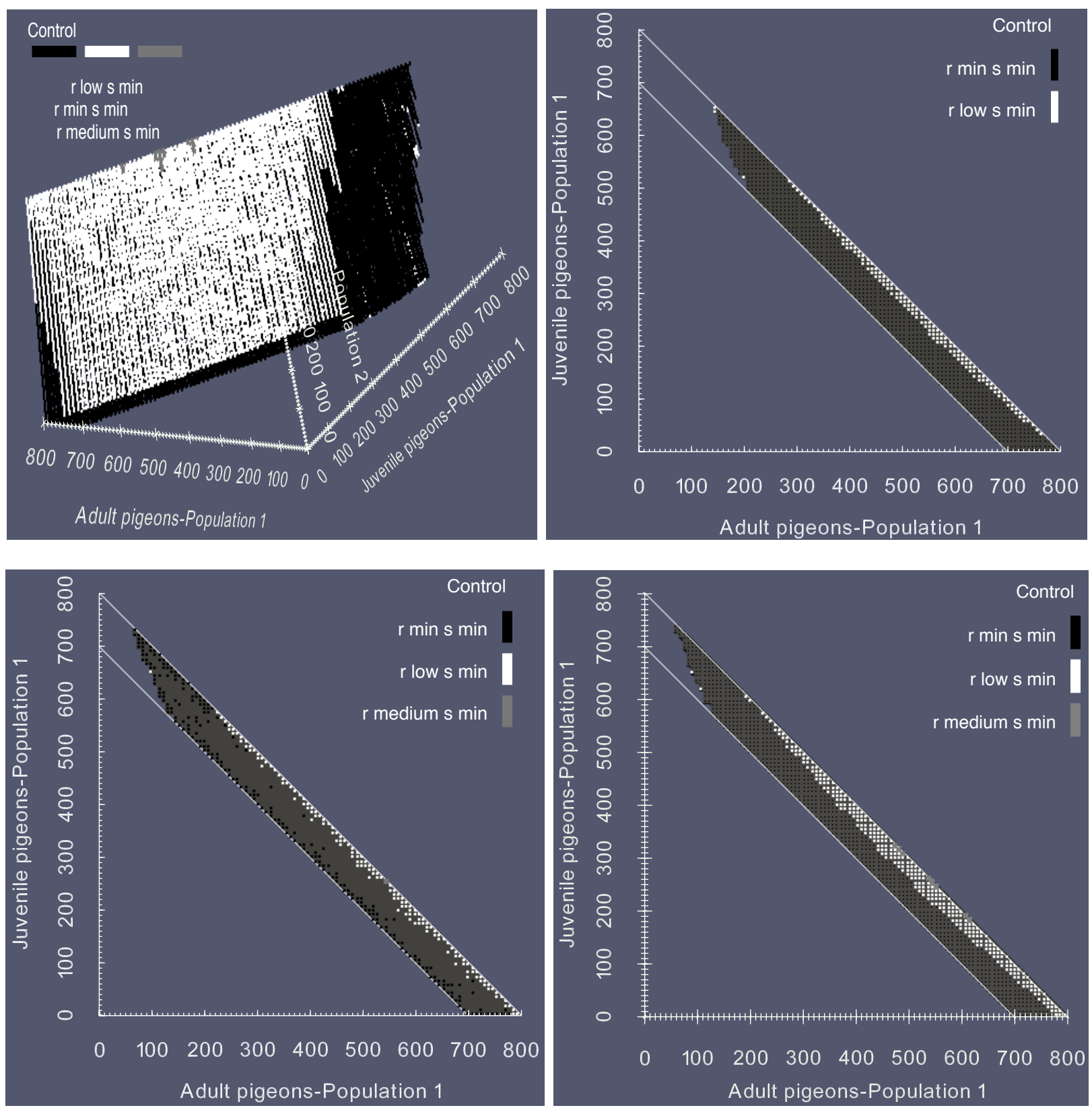

Figure 7: Figure left-top represents the viability kernel for the viability problem (32), in the case of the density dependent dispersal functions (34). Three clips at three different levels of the viability kernel: a first clip (right-top) for $x_{2}=0$, a second clip (left-bottom) for $x_{2}=450$ and the third clip (right-bottom) for $x_{2}=500$.

The choice of these two functions is given in oder to give a comparison between two situations: 1) constant dispersal rates and 2) density dependent dispersal rates respecting the fact that egg removal and limiting food resources encourage the dispersion of pigeons. The constraint set in this case is the one given by (13) with $\alpha_{1}=0.7, \beta_{1}=0.8, \alpha_{2}=0$ and $\beta_{2}=1$. By 
Figure 6 and Figure 7, we show the viability kernel in each case (case corresponding to (33) and (34)) as well as three clips at three different levels of the viability kernel: a first clip (right-top) for $x_{2}=0$, a second clip (left-bottom) for $x_{2}=450$ and the third clip (rightbottom) for $x_{2}=500$. The parameter values, concerning the constant dispersion rate, are chosen in the range of what is observed in Hetmański (2007) in the context of feral pigeon dispersion. In fact, in Hetmański (2007) the author shows that the dispersion among young birds is relatively high; it can reach $70 \%$ a year and remains at a stable level of 20 to $33 \%$ a year. As mentioned in section 2.1, the dispersion rate of adult pigeons is relatively low with respect to young pigeons, which justify the chosen values given by equation (33). We stress that this particular choice it is not at all unrepresentative. In fact, different constant dispersal rates, choosing in the range between 10 and $70 \%$ a year, show the same shape of the viability kernel.

The need to take into account the dispersal mechanism is analyzed here by comparing the viability kernel obtained in the case of one pigeon population with that obtained in the case of two pigeon populations. This is done by comparing Figure 5-right-bottom with Figure 6 and Figure 7. Two remarkable effects of the dispersal mechanism must be highlighted. The first one corresponds to the fact that the viability kernel becomes larger in the case of two pigeon populations; the second one corresponds to the fact that the control becomes more expensive in particular regions of the upper boundary. The expansion of the viability kernel can be simply justified by the fact that the arrival of new individuals allows the local population to avoid extinction when there are not enough adults, and this in accordance with Remark 2. The expansion of the viability kernel requires more expensive control in particular regions of the upper boundary of $K$. This can be simply justified by the fact that when the number of adult pigeons is sufficiently large, with the relatively high dispersal rate of the neighboring pigeons, the control strategy that worked well before (in the case of one pigeon population without dispersal effect) is not sufficient in this case, and this in accordance with Remark 2. In the light of these two remarks and after showing how a dispersal effect could modify the viability kernel, we can argue that any management strategy should take into account the dispersal mechanism within a pigeon population.

Remark 2. Remark, when the dispersal functions are given by equation (33), that for each $x_{2}=k$, with $k \in[0,500]$, and each $\alpha M \leq x_{j}, x_{a} \leq \beta M$ such that $x_{j}+x_{a}=\theta M$, with $\theta \in[\alpha, \beta]$, we have

$$
\dot{x}_{a}+\dot{x}_{j}=\Delta\left(x_{j}, x_{a}\right)+\underbrace{0.7 k+0.4 x_{a}-500 \theta}_{\Delta_{k}}
$$

where $\Delta\left(x_{j}, x_{a}\right)$ is given by equation (31).

Recall that equation (35) gives an idea about the velocity direction on each point $\left(x_{j}, x_{a}\right)$ such that $x_{j}+x_{a}=\theta M$ (and particularly on the boundaries of the constraint set $K$ when $\theta \in\{\alpha, \beta\})$ in the plane $x_{2}=k$, for $k \in[0,500]$. The quantity $\Delta_{k}$ can be seen as a perturbation of $\Delta\left(x_{j}, x_{a}\right)$. The positive sign of $\Delta_{k}$ allows to redirect the velocity in the upper boundary direction, while the negative one allows to redirect the velocity in the lower boundary direction. For example, remark that for $k=500$ and $\theta=\alpha$, we have that 
$\Delta_{k}=0.4 x_{a} \geq 0$. This explain the fact that in Figure 6-right-bottom the lower boundary of the viability kernel becomes larger. In addition, remark that for $k=500$ and $\theta=\beta$, we have $\Delta_{k}=0.4 x_{a}-50$. This explain, in Figure 6-right-bottom, the expansion of the upper boundary of the viability kernel in the region where there are not enough adults, and the need of more severe control in the region where the number of adult pigeons is sufficiently large.

\section{Conclusion and perspectives}

This work is an attempt at modeling the management of an urban pigeon population with an objective of cohabitation and not only a reduction of the pigeon population. Since it is not easy to describe precisely what should be the optimal population of pigeons regarding different factors, we have described the cohabitation problem in the framework of the mathematical theory of viability. Viability theory studies the compatibility between dynamical systems and state constraint sets. We have shown how the management of the pigeon population can be formulated as a viability problem: rather than an objective of population reduction, the objective is to maintain the state of the population in a set of desirable states. Urban citizens are supposed to be dissatisfied when the pigeon population exceeds a threshold because of the possible nuisance (this is a classical management viewpoint). Urban citizens are also supposed to be dissatisfied when the pigeon welfare is not ensured. Welfare could be represented by several state variables, for example the mean health state, or the lifetime. As a first approach, in order to keep a low number of state variables for computation reason, we have considered that the pigeon welfare can also be represented by the level of the total population: a low number of pigeons is a proxy a bad welfare conditions for the considered pigeon population. With this simplification hypothesis we were able to describe the set of desirable states of the pigeon population in terms of bounded levels of the total population. We have proposed an age structured model to describe the dynamics of the pigeon population with two age classes. In the viability framework, it was also necessary to consider explicitly the type of control actions in order to model their impact on the evolution of the pigeon population. So, we introduced control mechanisms with effects and parameters given by the literature, to model in a very simplified way egg removal and food control strategies. We have shown analytically some asymptotic properties of the dynamic, with no control and with constant control. In particular we have identified the equilibrium of the system and studied the phase portrait for some values of the control. From the viability viewpoint, even if the equilibrium belongs to the set of desirable states, some trajectories can still lead to non viable states, as this is the case in Figure 3 (right), and in that case the viability kernel can be smaller than the desirable sets. The computation of the viability kernel shows the advantage of adaptive control: the largest effort in term of control is only mandatory near the boundary of the viability kernel. Farther from the boundary no control effort is necessary, contrary to constant control policy. The viability study also confirms that the control actions are not adapted to ensure the pigeon welfare (this is not surprising, since the control aims only at diminishing the population level). In some cases (high juvenile population level with few adults), the evolutions are doomed to exit the set of desirable states whatever control 
is applied. We have also considered, in a simplified way, the possibility of spatial dispersion between two areas. After establishing the state of the art around the effects of egg removal and food control strategies on the dispersal mechanism, we integrated these effects in our model. We showed, by comparing the viability kernels calculated with spatial dispersion (see Figure 7 ) to that without spatial dispersion (see Figure 5), how this natural mechanism can modify the viability kernel and affects the controls. We have observed that the arrival of neighboring pigeons leads to strengthening the controls in particular region of the viability kernel. In addition, we observed that the dispersal mechanism leads to increase the viability kernel, meaning that this natural mechanism can play an important role to favor the cohabitation with the population of urban pigeons. Thus, while the arrival of neighboring pigeons can help the local population to avoid extinction, this might cost more to prevent the exceeding of a desirable threshold. Hence, this justifies the need for some regulatory programs, such as the egg removal or the limiting of food resources, in order to control the pigeon dispersion. This is an interesting message stating that, for a successful management strategy of an urban pigeon population, it is mandatory to take into account the dispersal mechanism.

The viability analysis, even performed on a simplified model, suggests interesting perspectives in term of management. The set of desirable states was set arbitrarily in this study, even if several set of values have been tested in order to present an overview of the possible situations. In order to challenge the viability framework in conditions closer to real-life, it would be necessary to build this constraint set with the stakeholders (local community council, local authority, etc.). Similarly, the choice of possible controls should be discussed with stakeholders, and one or several types of control with positive effect on pigeon welfare should be integrated in the model. Further work could also be done to exploit the possibilities in term of adaptive control: in this article two different controls could be used but with no information on their respective ratio cost vs. efficiency. Different control strategy could be derived a posteriori from the viability analysis. Since the viable states are given by the viability kernel, it would then possible to propose optimized strategy depending on criteria than can take the viability kernel into account (for example, switching from egg removal to resource control depending on costs, population level and distance to the boundary of the viability kernel). From the methodological viewpoint, further work is also needed to present viability analysis and results when the dimension of the state space is greater than three. This would have been the case here if we haven't simplified the dispersion between two areas model.

\section{Acknowledgment}

This work was partially funded by the DeMagma project of Convergence Program from Idex Super at Sorbonnes Universités.

\section{References}

AHRENS, J., GEVECI, B., LAW, C., 2005. Paraview: An end-user tool for large-data visualization. In: Hansen, C. D., Johnson, C. R. (Eds.), Visualization Handbook. Butterworth- 
Heinemann, Burlington, pp. $717-731$.

URL http://www.sciencedirect.com/science/article/pii/B9780123875822500381

Alvarez, I., de Aldama, R., Martin, S., Reuillon, R., 2013. Assessing the Resilience of SocioEcosystems: Coupling Viability Theory and Active Learning with kd-Trees. Application to Bilingual Societies. In: proceedings of IJCAI. pp. 2776-2782.

URL http://ijcai.org/papers13/Papers/IJCAI13-409.pdf

Alvarez, I., Martin, S., 2011. Geometric robustness of viability kernels and resilience basins. In: Deffuant, G., Gilbert, N. (Eds.), Viability and Resilience of Complex Systems. Understanding complex systems. Springer, pp. 193-218.

URL http://cemadoc.cemagref.fr/cemoa/PUB00033189

Alvarez, I., Reuillon, R., De Aldama, R., May 2016. Viabilitree: A kd-tree Framework for Viability-based Decision. Tech. rep., Irstea, preprint.

URL https://hal .archives-ouvertes.fr/hal-01319738

Aubin, J., 1991. Viability Theory. Birkhäuser, Basel.

Aubin, J.-P., Bayen, A., Saint-Pierre, P., 2011. Viability Theory: New Directions. Springer. URL http://hal .inria.fr/inria-00636570

Béné, C., Doyen, L., Gabay, D., 2001. A viability analysis for a bio-economic model. Ecological Economics 36 (3), 385 - 396.

Bernard, C., Martin, S., 2013. Comparing the sustainability of different action policy possibilities: Application to the issue of both household survival and forest preservation in the corridor of fianarantsoa. Mathematical Biosciences 245 (2), 322 - 330.

Boulinier, T., McCoy, K. D., Yoccoz, N. G., Gasparini, J., Tveraa, T., 2008. Public information affects breeding dispersal in a colonial bird: kittiwakes cue on neighbours. Biol. Lett. $4,538-540$.

Falcone, M., Saint-Pierre, P., 1987. Slow and quasi-slow solutions of differential inclusions. Nonlinear Analysis: Theory, Methods \& Applications 11 (3), 367-377.

Giunchi, D., Baldaccini, N. E., Sbragia, G., Soldatini, C., 2007. On the use of pharmacological sterilization to control feral pigeon populations. Wildlife Research 34, 306-318.

Greenwood, P. J., Harvey, P. H., 1982. The natal and breeding dispersal of birds. Ann. Rev. Ecol. Syst. 13, 1-21.

Haag, D., 1991. Population density as a regulator of mortality among eggs and nestlings of feral pigeons (columba livia domestica) in basel, switzerland. In: Proceedings of the International Symposium of the Working Group on Granivorous Birds, INTECOL, Slupsk, Poland, Sept 14-17 1989, Warzawa: J. Pinowski, B. P. Kavanagh and W. Gorski, 21-31. 
Haag-Wackernagel, D., 1993. Street pigeons in basel. Nature 361, 200.

Haag-Wackernagel, D., 2002. Feral pigeons: management experiences in europe atti 2 convegno nazionale sulla fauna urbana "specie ornitiche problematiche: biologia e gestiona nelle città e nel territorio", firenze, 10 giugno 2000. ARSIA e LIPU. Firenze. M. Dinetti, Regiona Toscana, 25-37.

Haag-Wackernagel, D., Heeb, P., Leiss, A., 2006. Phenotype-dependent selection of juvenile urban feral pigeons columba livia: We suggest the existence of colour-based selection processes on juvenile feral pigeons in an urban environment. Bird Study 53 (2), 163-170.

Harvey, P. H., Greenwood, P. J., Perrins, C. M., 1997. Breeding area fidelity of the great tit (parus major). J. Anim. EcoL 48, 305-313.

Hetmański, T., 2007. Dispersion asymmetry within a feral pigeon columba livia population. Acta Ornithol. 42 (1), 23-31.

Hinde, R. A., 1952. The behaviour of the great it (Parus major) and some other related species. E.J. Brill.

Jacob, G., Prévot-Julliard, A., Baudry, E., 2014. The geographic scale of genetic differentiation in the feral pigeon (columba livia): implications for management. Biological Invasions doi: 10.1007/s10530-014-0713-2.

Jacquin, L., Cazelles, B., Prévot-Julliard, A. C., Leboucher, G., Gasparini, J., 2010. Reproduction management affects breeding ecology and reproduction costs in feral urban pigeons (columba livia). Canadian Journal of Zoology 88, 781-787.

Jerolmack, C., 2008. How pigeons became rats: the cultural-spatial logic of problem animals. Social Problems 55, 72-94.

Kautz, J. E., Malecki, R. A., 1991. Effects of harvest on feral rock dove survival, nest success and population size. Department of the Interior, Fish and Wildlife Service, Washington, DC.

Lara, M. D., Martinet, V., 2009. Multi-criteria dynamic decision under uncertainty: A stochastic viability analysis and an application to sustainable fishery management. Mathematical Biosciences 217 (2), 118 - 124.

Murton, R. K., Thearle, R. J., Coombs, C. F. B., 1974. Ecological studies of the feral pigeon columba livia var. iii. reproduction and plumage polymorphism. Journal of Applied Ecology 11 (3), 841-854.

Récapet, C., Dauphin, L., Jacquin, L., Gasparini, J., Prévot-Julliard, A. C., 2006. Phenotype-dependent selection of juvenile urban feral pigeons columba livia: We suggest the existence of colour-based selection processes on juvenile feral pigeons in an urban environment. Bird Study 53 (2), 163-170. 
Rouquier, J.-B., Alvarez, I., Reuillon, R., Wuillemin, P.-H., 2015. A kd-tree algorithm to discover the boundary of a black box hypervolume. Annals of Mathematics and Artificial Intelligence, $1-16$.

URL http://dx.doi.org/10.1007/s10472-015-9456-8

Sacchi, R., Gentilli, A., Razzetti, E., Barbieri, F., 2002. Effects of building features on density and flock distribution on feral pigeons columba livia var. domestica in an urban environment. Canadian Journal of Zoology 80, 48-54.

Skandrani, Z., Daniel, L., Jacquelin, L., Leboucher, G., Bovet, D., Prévot, A. C., 2015. On public influence on people's interactions with ordinary biodiversity. PLoS One. URL http://dx.doi.org/10.1371/journal.pone.0130215

Skandrani, Z., Lepetz, S., Prévot-Julliard, A., 2014. Nuisance species: beyond the ecological perspective. Ecological processes.

URL http://www. ecologicalprocesses.com/content/3/1/3 